\title{
TV/Series
}

1 | 2012

Les Séries télévisées américaines contemporaines :

entre la fiction, les faits, et le réel

\section{Les figurations du corps dans Deadwood: ancrage historique, réalisme et théâtralité}

\section{Gilles Menegaldo}

\section{(2) OpenEdition}

\section{Journals}

Édition électronique

URL : http://journals.openedition.org/tvseries/1547

DOI : 10.4000/tvseries. 1547

ISSN : 2266-0909

Éditeur

GRIC - Groupe de recherche Identités et Cultures

Référence électronique

Gilles Menegaldo, «Les figurations du corps dans Deadwood : ancrage historique, réalisme et théâtralité », TV/Series [En ligne], 1 | 2012, mis en ligne le 15 mai 2012, consulté le 19 avril 2019. URL http://journals.openedition.org/tvseries/1547; DOI : 10.4000/tvseries.1547

\section{(c) (i) (9)}

TV/Series est mis à disposition selon les termes de la licence Creative Commons Attribution - Pas d'Utilisation Commerciale - Pas de Modification 4.0 International. 


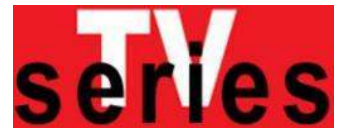

\title{
Les figurations du corps dans Deadwood : ancrage historique, réalisme et théâtralité
}

Gilles MENEGALDo,

\begin{abstract}
Deadwood a réellement existé ainsi que certains personnages centraux et bon nombre de personnages secondaires. Certains sont des icônes de l'ouest mythique (Wild Bill Hickok, Calamity Jane), d'autres sont également des figures historiques comme Al Swearengen, le patron du «Gem saloon » ou encore George Hearst, père de William Randolph Hearst. La mise en fiction repose sur la transformation de personnages réels ou l'invention de nouveaux personnages comme la petite Sophia, ultime rescapée d'un massacre réel ou encore Alma Garret qui fait l'objet de différents enjeux. Le démarquage de la réalité se fait aussi par l'emploi d'un riche réseau intertextuel littéraire, cinématographique et philosophique.

Outre la représentation du corps mythifié, la série se caractérise par une vision très réaliste du corps placé souvent dans des situations extrêmes. Le corps masculin ou féminin est un objet de désir, et un enjeu économique ou politique. La violence est un mode dominant et les femmes, exploitées sexuellement, en proie à la maladie et à l'emprise des drogues en sont les victimes privilégiées. Cette violence s'exprime par la récurrence des meurtres et des scènes de bagarre mais aussi par la mise en scène du corps souffrant. Le corps s'inscrit dans un mode de figuration grotesque qui met l'accent sur l'exagération ou la distorsion des traits physiques et privilégie une esthétique de la laideur. Les activités corporelles les plus intimes sont évoquées afin de privilégier la dimension sordide et dérisoire de l'existence au détriment d'une approche romantique dont il reste quelques traces.

Le corps est cependant dramatisé par sa relation privilégiée au langage qui participe d'un effet de réel mais contribue aussi à théâtraliser certaines scènes par l'emploi de dialogues qui relèvent d'une langue littéraire métaphorique et enrichie par des emprunts shakespeariens. C'est la parole qui redonne une forme de noblesse ou un sens du tragique à des personnages dont le corps est souvent trivialisé ou réifié.
\end{abstract}

$\mathrm{D}$ eadwood, petite ville minière rattachée au Dakota en 1876 a réellement existé ainsi que certains personnages centraux et bon nombre de personnages secondaires mis en scène dans la série télévisée éponyme. David Milch, créateur de cette série HBO, exploite ce moment emblématique de l'histoire de l'Ouest, de l'extension de la frontière, pour confronter différents systèmes de valeurs et en particulier les notions d'ordre et de loi. L'ancrage au réel historique est donc patent et la série réalisée entre 2004 et 2006 et qui comporte trois saisons ( 36 épisodes de $50 \mathrm{mn}$ ) vise à montrer la transformation graduelle de ce mining settlement, au départ simple campement dont l'implantation sur les terres des Indiens Lakota proches des Black Hills, est illégale, et qui va obtenir le rattachement (annexation) aux États-Unis d'Amérique. Après un prologue situé dans le Montana et mettant en scène le marshal Seth Bullock (Timothy 
Oliphant) procédant à la pendaison d'un vendeur de chevaux pour éviter son lynchage, la série s'ouvre sur un plan large d'une caravane de pionniers, au moment où une nouvelle vague de chercheurs d'or arrive à Deadwood et alors que les tentes sont déjà largement remplacées par des structures plus solides en bois.

Certains protagonistes sont des icônes de l'ouest mythique comme Wild Bill Hickock, ou Martha Jane Canary ou encore les frères Earp qui font une apparition dans la troisième saison. D'autres moins connus du public sont également des figures historiques comme $\mathrm{Al}$ Swearengen, le patron du "Gem saloon », Seth Bullock, construit en héros a priori plus positif, son partenaire en affaires Sol Star, E.B. Farnum, l'hôtelier "nommé » maire de la ville, ou encore George Hearst, père du célèbre William Randolph Hearst immortalisé par Orson Welles et qui devient une figure centrale dans la $3^{\mathrm{e}}$ saison. Deadwood se fonde donc sur une réalité historique précise, même si l'imaginaire de David Milch se nourrit aussi de westerns hollywoodiens et en reprend certains lieux emblématiques (la rue, le saloon) mais aussi certains topoï comme les duels, les bagarres, les scènes de jeu. À cet égard, la série relève sans doute davantage du western révisionniste ou anti-western, de par son approche critique, ironique et désenchantée par rapport à la mythologie westernienne. Il n'y a pas à proprement parler de héros positif et le personnage principal Al Swearengen qui dirige son établissement (un saloon mais aussi un bordel) d'une main de fer, et commandite divers meurtres, est loin d'incarner les valeurs chevaleresques du héros westernien incarné par James Stewart dans les films d'Anthony Mann ou Gary Cooper dans High Noon de Fred Zinnemann.

La mise en fiction repose sur la transformation plus ou moins marquée des personnages réels. Al Swearengen (Ian McShane) voit son rôle assez largement amplifié et il occupe l'espace filmique d'un bout à l'autre de la série, son statut et son image évoluant sensiblement. Au début il est caractérisé comme un «bad guy » violent et manipulateur, cruel et cynique, mais aussi un homme d'envergure au charisme certain, entouré d'êtres (hommes et femmes) à sa dévotion, et doté d'un sens politique aigu. Ces qualités ${ }^{1}$, perceptibles dès le départ, lui permettent d'acquérir une autre stature, de s'humaniser et de devenir une sorte de figure protectrice de la communauté tout en conservant sa

\footnotetext{
${ }^{1}$ Le Swearengen fictionnel est calqué en partie sur le personnage historique qui arrive à Deadwood en 1876 et ouvre dès 1877 le Gem Variety Theatre qui en effet comme dans la série était surtout un lieu de prostitution (et de trafics divers) mais qui présentait aussi des spectacles de variété. Le Swearengen réel s'enrichit rapidement (grâce aussi au jeu et à la drogue) et sa gestion très brutale des prostituées et diverses rixes lui valent sa réputation. Marié et divorcé à 3 reprises (alors qu'il est célibataire dans la série), Swearengen ruiné quitte Deadwood en 1899 et meurt assassiné en 1904 à Denver. Milch transforme assez profondément ce personnage et lui donne un sens politique et surtout un charisme dont le vrai Swearengen était sans doute dépourvu. Voir l'ouvrage d'Elizabeth Fifer, Bad Boys of the Black Hills, Farcountry Press, 2008.
} 
volonté de pouvoir et de contrôle. Il s'oppose à la volonté hégémonique et la brutalité de Georges Hearst qui arrive en conquérant et devient un personnage essentiel dans la troisième saison. Comme le dit Paul A. Cantor : "Swearengen emerges as the chief architect of order without law in Deadwood $»^{2}$. Swearengen est aussi le focalisateur principal, le sujet d'un regard qui se veut panoptique et qui souvent ouvre et conclut tel ou tel épisode. Il est filmé fréquemment en position d'observateur privilégié, en surplomb, campé fermement sur sa terrasse ou derrière sa fenêtre, saisi en contre plongée ou en plan semi subjectif, le dos tourné à la caméra. Seul George Hearst aura droit à ce regard dominant, en particulier dans la troisième saison où les deux rivaux s'affrontent du regard de part et d'autre de la rue centrale.

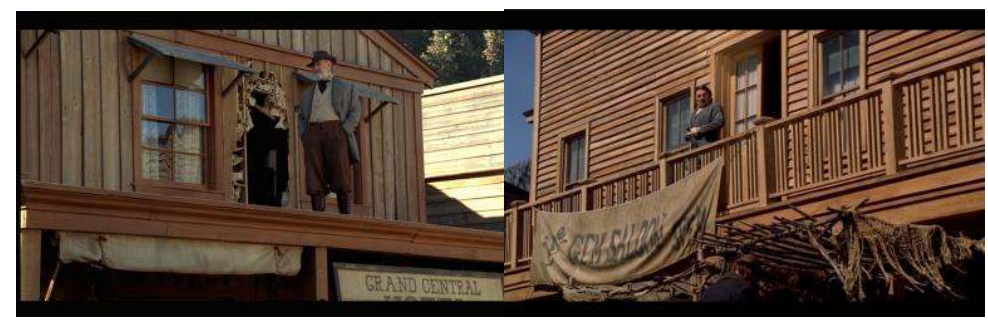

Cette force du regard est cependant ironiquement atténuée du fait que Swearengen dont la vue baisse doit se servir d'une loupe pour lire les différents documents qui transitent de manière licite ou illicite dans son bureau. David Milch invente de nouveaux personnages comme la petite Sofia, ultime rescapée d'un massacre réel ou encore Alma Garret, venue de l'Est avec Brom son riche mari et qui fait l'objet de différents enjeux (sentimentaux mais surtout financiers) tout au long de la série. Surtout, il modifie notablement le rôle historique de tel ou tel personnage. C'est le cas pour Hearst qui est particulièrement diabolisé. Le démarquage de la réalité se fait également par l'emploi d'un riche réseau intertextuel littéraire, cinématographique, mais aussi philosophique (Hobbes, Locke, Rousseau 3 ).

Le générique très élaboré est constitué d'un montage alterné qui nous montre d'une part la course d'un étalon dans différents cadres bucoliques et sauvages, et d'autre part divers lieux et diverses activités humaines à Deadwood (campement boueux et homme dormant à même le sol, bouchers découpant de la viande, carcasses de porcs et de poulets, chercheur d'or aux dents cariées, filmé de profil, mordant une pépite, femme nue de dos dans une baignoire, jeu de cartes...). Le très

2 Paul A. Cantor, « Deadwood and the State of nature », The Philosophy of the Western, éd. Jennifer L. McMahon et B. Steve Csaki, Lexington, University Press of Kentucky, 2010, p. 124 .

3 Voir aussi sur ce point l'article de Cantor, op. cit. 
gros plan d'un sabot du cheval s'enfonçant dans la boue traduit la puissance de l'animal mais annonce aussi le rôle tragique qu'il jouera dans le destin d'une famille. Deux activités se succèdent ensuite et se font écho : une main hors champ verse de l'alcool, puis la poussière d'or s'écoule. La juxtaposition des deux plans suggère la transaction commerciale qui est au cœur de l'activité de Deadwood.

Christophe Chambost 4 fait une lecture métaphorique séduisante de la fin du générique :

Enfin, on ne voit plus que le reflet du cheval dans une flaque d'eau : l'animal avance au pas dans la rue principale de Deadwood, et son reflet disparaît comme par magie (ou plutôt, comme dans un cauchemar) alors que le reflet du « Gem saloon » reste sur l'écran jusqu'au fondu au noir final. Le message semble clair : au contact de la bassesse humaine, la nature s'évanouit et laisse place au lieu de tentation et de perdition qu'est le bordel d'Al Swearengen, plus tard dédoublé par celui de $\mathrm{Cy}$ Tolliver, la Bella Union.

Ce montage d'images met déjà en relief certains des motifs dominants de la série, notamment la relation entre la nature libre et sauvage et la civilisation, mais aussi la représentation du corps dans son environnement et dans sa relation avec l'animalité.

Je souhaite me concentrer sur cette mise en scène du corps, un des aspects les plus frappants de la série. Cette question de la représentation de l'organique, centrale dans l'univers diégétique, va de pair avec la violence de l'univers représenté, celle qui régit la vie quotidienne d'une communauté qui se constitue en l'absence de tout pouvoir institutionnel légal et en fonction d'intérêts personnels dictés par la volonté de contrôle et d'appropriation des territoires et des corps, et la satisfaction des désirs matériels mais aussi des obsessions monomaniaques et des fantasmes plus ou moins pervers des individus. Dans ce monde sans loi, mais non dépourvu d'ordre, le corps occupe une place primordiale, à la fois locus dynamique, mais fragile, objet de désir et de transaction, enjeu dramatique et symbolique.

La représentation du corps se décline de manière diversifiée. Le corps est ainsi mythifié comme celui, dans les premiers épisodes, de Wild Bill (Keith Carradine, impérial) avec tous ses attributs physiques (cheveux longs, moustache tombante, regard hautain) et vestimentaires (veste en peau effrangée, chapeau, assez fidèlement reproduits par rapport aux portraits et photographies existants)

4 Voir sa communication au colloque de Cerisy Le Western et les mythes de l'ouest, « La fange et le filon, ou comment Deadwood ré-investit le western ». À paraître aux PU Rennes en 2012. 

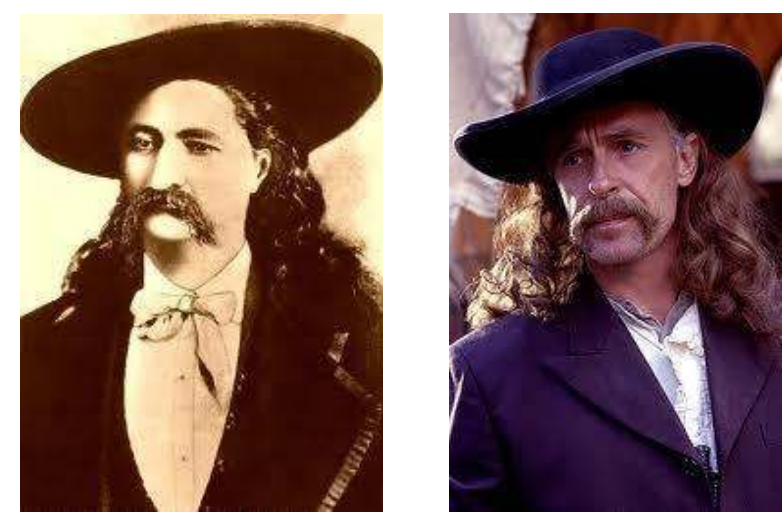

ou démystifié comme Calamity Jane (Robin Weigert), anti-héroïne androgyne, alcoolique et froussarde, entre propos orduriers et bravaches et crises de larmes. La scène où elle ne peut empêcher $\mathrm{Al}$ de pénétrer dans la chambre de Sofia la petite fille pour voir si elle vit encore - il lui pince assez violemment le poignet - souligne son impuissance totale face au pouvoir patriarcal du patron du Gem Saloon. Calamity Jane fut en réalité la première femme blanche à pénétrer dans les « Black Hills », un territoire du Dakota du Sud, dans les montagnes sacrées contrôlées par les Sioux.

La présence corporelle s'exprime par le surpeuplement, le grouillement, la saturation du cadre envahi par les corps, les regroupements de foule tant à l'extérieur dans la rue principale, qu'à l'intérieur (dans les saloons). La série se caractérise par une mise en scène très réaliste du corps placé souvent dans des situations extrêmes. Le corps masculin ou féminin est un objet de désir soumis à la violence légale ou illégale, mais aussi un enjeu économique ou politique.

La violence est un mode dominant dans une communauté où la vie humaine a peu de prix, d'où la dimension darwinienne et « hobbesienne » de la série. Al Swearengen mais surtout George Hearst peuvent en effet être assimilés à une figure de Léviathan (un des épisodes s'intitule «Leviathan Smiles »). Dès le début de la première saison, la souffrance infligée au corps est mise en relief dans la scène de pendaison du voleur de chevaux, scène emblématique de nombreux westerns. L'acte est perpétré par un personnage incarnant la loi et qui abrège l'agonie du condamné en lui brisant le cou. Seth Bullock quittera ensuite le Montana pour s'installer à Deadwood afin d'ouvrir un commerce de quincaillerie (hardware) avec son partenaire Sol Star, seul personnage identifié comme juif (et en butte à divers propos antisémites) dans la série. Seth et Sol ont compris qu'il y avait de l'argent à gagner en fournissant aux prospecteurs l'équipement nécessaire. 
Un peu plus tard, une prostituée, Trixie (dont le rôle est essentiel à bien des égards dans l'ensemble de la série), tue d'une balle dans l'œil un client qui la brutalisait. Outre le visage tuméfié de la femme, la caméra donne à voir en gros plan graphique, à la limite du gore, l'homme affalé agonisant, la respiration de plus en plus difficile, et le trou béant dans sa tête d'où sourd le sang. Près du Gem Saloon se trouve aussi le quartier chinois où le linge est lavé et les cadavres éliminés par des moyens radicaux, mais peu orthodoxes. Le blanchissage, activité emblématique de la communauté chinoise acquiert une dimension organique. Le cadavre du client brutal sert dans une scène ultérieure de nourriture aux cochons voraces de monsieur $\mathrm{Wu}$, un chinois énigmatique qui contrôle le quartier et le trafic de drogue. Ce recyclage naturel qu'on voit s'opérer à plusieurs reprises dans la série permet de faire disparaître discrètement les cadavres, mais montre aussi que la vie humaine a peu de prix. Bien plus tard (2.11), les cochons sont supplantés par une autre méthode de suppression des corps, puisque les cadavres des prostituées chinoises sont brûlés en pleine rue.

D'emblée donc le corps est mis en exergue, le camp en est le locus horribilis. Les prospecteurs qui arrivent en chariot, saisis selon le point de vue de Calamity Jane dans un lent panoramique latéral qui offre une première vision du settlement rudimentaire, passent leurs journées sur leur concession et reviennent au village pour soigner leur corps fatigué ou meurtri. Ils prennent des bains pour éliminer la saleté, ils se nourrissent, boivent et se livrent à des activités sexuelles, d'où le rôle clef du Gem saloon, pourvoyeur des prostituées que l'on découvre dès le premier épisode sur un balcon, leurs visages dissimulés sous des ombrelles, première image trompeuse vite démentie par la scène de meurtre d'un "client " évoquée plus haut et suivie d'une scène non moins violente où $\mathrm{Al}$ Swearengen brutalise Trixie (Paula Malcomson) appuyant sa botte sur son cou meurtri jusqu'à l'étouffer pour s'assurer de sa soumission. L'autre scène traumatique au début de la série concerne le massacre par des bandits de grand chemin (plus ou moins associés à Swearengen) d'une famille de pionniers norvégiens, les Metz, qualifiés avec mépris de "squareheads » et dont les corps sont en partie démembrés. Lors d'une expédition nocturne, les cadavres partiellement dévorés par les loups sont découverts. Les plans sur les corps mutilés sont brefs mais très explicites. Seule la petite Sofia échappe au massacre et sa survie va constituer l'un des enjeux des débuts de la série. Ce premier épisode se conclut par un affrontement meurtrier au cours duquel Wild Bill tue d'une balle dans l'œil (écho au meurtre précédent) Ned Mason, un des bandits (road agent) suspecté de participation au massacre de la famille. Il tuera ensuite Tom Mason son frère, chargé par $\mathrm{Al}$ de l'éliminer.

Dès les trois premiers épisodes, les morts violentes s'accumulent : pendaison, meurtre au couteau, crâne fracassé (celui de 
Barrett tué par Dan Dority, exécuteur des basses œuvres de Swearengen), œil perforé par une balle (à deux reprises), gorge tranchée etc. La série multiplie ensuite les scènes de violence et d'affrontement physique. Parmi les plus mémorables, le combat à mort entre Seth Bullock qui a par mégarde profané un lieu sacré et un indien Lakota dont il écrase la tête à coups de pierre, les tortures physiques et la mort violente endurées par les jeunes adolescents Flora et Miles Anderson (clin d'œil au Turn of the Screw d'Henry James) déjà corrompus malgré leur jeune âge. Ils ont tenté de voler Cy Tolliver qui se venge en les frappant sauvagement avant de les exécuter, sous le regard effaré de Joannie Stubs, la « madame » de la Bella Union. Al et Seth s'affrontent aussi physiquement au début de la saison deux, Seth ne supportant pas les allusions d'Al à sa relation avec Alma et $\mathrm{Al}$ reprochant à Seth de s'occuper plus de ses affaires privées que de son travail de sheriff, comme l'atteste cette apostrophe non dénuée de sous-entendus : " Or, with you at her ear, among other points of entry, instead of doing your civic duty ». Le corps à corps est spectaculaire, la caméra épousant de près les gestes des combattants. Au moment où Seth a le dessus, Dority l'assomme d'un coup de crosse, redonnant l'avantage à $\mathrm{Al}$, qui contre tout fair play sort son couteau et s'apprête à achever Seth. Seul le regard du jeune William Bullock qui contemple la scène depuis l'intérieur de la diligence qui vient d'arriver à Deadwood parvient à stopper le geste mortel. Comme le dit Swearengen, filmé en gros plan, le regard orienté vers la caméra et donc prenant le spectateur à témoin: "The cow-eyed kid looking from that coach, that's what fucking unmanned me ».

L'épisode Two Headed Beast met en scène le combat plus épique encore entre Dan Dority et le capitaine Turner, la brute qui fait office de champion de Hearst. En guise de prologue on voit Dan face caméra s'enduire le corps d'huile et Turner s'entraîner devant son patron. L'affrontement se déroule en pleine rue, en public, et sous le regard des deux opposants, Swearengen et Hearst qui se font face, campés sur leurs balcons respectifs. Le combat est montré de différents points de vue, l'angle de la caméra, portée et instable, variant constamment ainsi que la distance. La caméra est parfois tout proche des corps, parfois beaucoup plus éloignée, permettant une vision d'ensemble, englobant les combattants et le public. Le montage est serré, rythmant les différentes phases du combat et la bande sonore (bruits des corps en mouvement ou en contact, halètements sourds, gémissements, hurlements de douleur etc.) met aussi en relief la dimension organique. Les corps s'effondrent parmi les quartiers de viande, rampent et s'enfoncent dans la boue. Turner hurle quand Dan, couvert de sang, lui arrache sauvagement un œil et, filmé en contreplongée en héros victorieux, l'achève d'un coup de bûche comme un animal, obtenant un signe à peine perceptible d'assentiment de son employeur, situé au-dessus de lui. 
Même les transactions commerciales sont liées au corps. Ainsi Brom Barrett, le premier mari d'Alma, et le prospecteur crachent dans leurs mains pour sanctionner la vente de la mine d'or qui sera l'un des enjeux financiers principaux tout au long de la série. Les pionniers, clients des deux saloons, sont caractérisés par leurs odeurs corporelles lors d'une conversation entre $\mathrm{Al}$ et $\mathrm{Cy}$ Tolliver, le rival qui vient de s'installer :

Cy. If you are a pioneering type, a trailblazing type, you are gonna draw a trailblazing element.

AL. ...meaning I get the ones that don't wash.

$\mathrm{Cy}$. We must cut through the stink though when they walk in with those sacks full of gold.

Les références aux fluides corporels sont très nombreuses et ce dès les premiers épisodes. Les hommes urinent en privé ou en public (Al dans un pot, Charlie Utter à même le sol, un homme meurt d'avoir uriné sur un autre et souillé ses vêtements...), salivent, expectorent, vomissent. Alma Garrett, femme raffinée, ne peut non plus s'empêcher de vomir alors qu'elle vit une grossesse difficile. Farnum se fait tabasser sévèrement par Bullock, mais aussi de manière plus humiliante cracher à la figure en gros plan par Hearst qui lui interdit de s'essuyer le visage sous peine de mort. Farnum obéit pendant un temps, puis dans un accès de révolte dérisoire, finit par éponger la salive, imaginant dans un monologue très théâtral une situation encore plus dégradante: "There remains receiving his regurgitations or swallowing his faeces ». Farnum souffre également de problèmes gastriques tout comme Harry Manning, le boulimique et peu glorieux candidat au poste de sheriff. Le sang est le fluide le plus visible, il s'écoule des nombreux corps blessés ou tués à l'arme blanche ou au revolver, jaillit des gorges tranchées par Swearengen ou ses acolytes, mais aussi Francis Wolcott, géologue à la solde de Hearst et maniaque sexuel et tueur au rasoir de prostituées (sa "carrière » commence avant Deadwood). Al, grand «purificateur» passe son temps à nettoyer les taches de sang laissées par les corps sur le parquet de son bureau ou celui du saloon, théâtre de plusieurs meurtres. La série se termine symboliquement sur un dernier nettoyage, celui du sang de Jen, jeune prostituée faisant office de «vierge sacrifiée» pour satisfaire le désir de vengeance de Hearst, mais surtout pour lui donner le change et sauver la vie de Trixie, le véritable auteur de la tentative de meurtre du magnat.

Les mauvaises odeurs corporelles sont également évoquées verbalement (Al menace de jeter dehors celui qui émettrait des vents) ou suggérées visuellement (sueur, crasse des vêtements etc.). Trixie, passant en revue les prostituées juste avant l'enterrement de William Bullock, apostrophe Jen en termes crus, lui reprochant sa mauvaise 
haleine : «And wash your fucking mouth. You got seven kinds of cock breath ». Les corps sont aussi très souvent en contact avec la matière, en particulier la poussière et la boue omniprésente dans la rue. Les activités corporelles les plus intimes sont mises en scène de manière réaliste, la sexualité, mais aussi d'autres aspects moins souvent représentés à l'écran. Ainsi, la défécation est évoquée au moins verbalement à plusieurs reprises et le mot « shit » est utilisé à la fois littéralement et métaphoriquement («shitface» est une insulte récurrente).

Les scènes sexuelles sont souvent amorcées mais rarement mises en scène en détails et en gros plan. La caméra reste à distance des corps relégués au fond du cadre et/ou en partie cachés par des éléments de décor. La bande sonore, faite de grincements rythmiques de matelas, de cris, de râles de jouissance remplace fréquemment l'image. La mise en scène met l'accent sur les prémisses de la relation ou ce qui se passe après l'acte. Une des rares exceptions à ce parti pris concerne l'unique scène d'amour filmée entre Seth Bullock et Alma et dont on voit trois moments : un plan moyen en caméra fixe filme les ébats sexuels, un peu plus tard, la caméra se rapproche jusqu'au gros plan sensuel des corps nus enlacés au moment de l'orgasme, enfin une scène plus longue montre le couple apaisé après l'amour, sans doute l'unique scène d'intimité affective entre les deux amants éphémères. À cet exemple près, l'érotisme est assez rare dans une série où la sexualité est souvent présentée sous ses dehors sordides, voire obscènes ou abjects ou comme un acte mécanique dénué d'affect ou encore sous la forme d'une relation sadique et mortifère.

La nudité est cependant bien présente, en particulier celle des femmes, principales victimes des désirs plus ou moins pervers des hommes et réduites au statut d'objet sexuel. Trixie, la prostituée «favorite" de $\mathrm{Al}$ dont le rôle est privilégié dans la série car elle interagit avec les différents clans, ôte ses vêtements avant de se glisser dans le lit et de faire l'amour avec son maître (on ne voit pas la scène) qui profite aussi de la dextérité de Dolly, une jeune prostituée qui lui dispense régulièrement des fellations (blow jobs) avec plus ou moins d'habileté. D’autres prostituées se dénudent soit dans l'intimité du " gynécée », soit pour s'exposer au regard des clients et les aguicher. Trixie dévoile ses seins de manière spectaculaire (comme une héroïne antique ou une pasionaria révolutionnaire) au moment où elle tente de tuer Hearst. Le corps masculin est aussi montré nu, mais plus rarement et ce afin d'accentuer la dimension bestiale du personnage. C'est ainsi le cas de Tom Mason le bandit qui avance en pleine lumière, nu et le pénis érigé, éructant une obscénité : "this snatch is branded ! ». Cette «full frontal nudity» est assez rare pour être remarquée.

Il s'agit bien de privilégier la dimension cruelle, mais aussi sordide et dérisoire de l'existence au détriment d'une approche 
romantique dont il ne reste que quelques traces, en particulier dans la relation amoureuse passionnée mais éphémère qui s'instaure entre Seth et Alma Garrett avant le remariage de celle-ci avec le prospecteur Ellsworth qui incarne, contrairement au premier mari faible et féminisé, une certaine forme de virilité et de responsabilité.

Les hommes n'échappent pas à la violence faite au corps qui s'exprime par l'exploitation de la force de travail dans les mines de Hearst, par la récurrence des crimes affectant aussi bien les hors la loi que les ouvriers (syndicalistes poignardés sur ordre du magnat). Le rapport étroit entre vie et mort est figuré par le « recyclage» des cadavres effectué par Swearengen, et par les scènes de bagarre plus ou moins ritualisées, mais aussi par la mise en scène du corps souffrant. Les blessures sont variées : au bas ventre, en haut de la cuisse, au cou, à la tête. Les tabassages sont fréquents : Bullock démolit à coups de poing le visage de Swearengen, puis de Farnum, Charlie Utter qui a appris la vérité sur les meurtres de prostituées tabasse violemment Wolcott, un Pinkerton frappe brutalement le journaliste Merrick pour le punir d'avoir imprimé une lettre de Bullock qui met indirectement en cause Hearst dans l'affaire du meurtre d'un ouvrier de sa compagnie. Même les officiels ne sont pas à l'abri. Ainsi, $\mathrm{Al}$ fait couper la gorge d'un envoyé de Yankton, le magistrat Claggett, politicien douteux du Sud Dakota et roue de coups un agent Pinkerton, ancien soldat, avant de l'égorger. Cy Tolliver est victime de la vengeance d'Andy Cramed, l'ancien compagnon qu'il a abandonné, atteint de la variole. Celui-ci devenu pasteur est plusieurs fois brutalement expulsé de la Bella Union sur ordre de Cy qu'il finit par poignarder, à la grande surprise de sa victime. Au cours du dernier épisode, Cy frustré et humilié par Hearst qui lui exprime son mépris, blesse à son tour gravement Leon, l'un de ses fidèles séides qui s'écroule, stupéfait en se tenant le bas-ventre. La violence concerne aussi l'automutilation, ce que reproche le docteur à Tolliver, ou les nombreuses tentatives de suicide. Trixie et Joannie ne passent pas à l'acte mais Hostetler le palefrenier noir, excédé par le comportement hystérique de son antagoniste blanc, Steve, braillard alcoolique et viscéralement raciste, se donne la mort de manière inattendue.

Un exemple emblématique de cette souffrance corporelle qui raidit les corps et crispe les visages est celui de l'opération pratiquée sur $\mathrm{Al}$ qui souffre de calculs rénaux très douloureux. Cette scène un peu traumatique pour le spectateur contribue à humaniser le personnage qui souffre ainsi dans sa chair. On le voit d'abord incapable de parler et d'appeler à l'aide, le corps recroquevillé sur le sol, agité de tremblements. Quand le Docteur Cochran (Brad Dourif) parvient à le convaincre de se faire soigner, $\mathrm{Al}$ subit une intervention très pénible (une aiguille traverse son pénis) visant à localiser le calcule. Il échappe par miracle à une opération encore plus risquée et se remet lentement d'une paralysie partielle, conséquence d'une attaque cérébrale. Plus 
tard, lors de la troisième saison, un de ses doigts est sectionné (castration symbolique) par George Hearst qui par ce coup de marteau affirme un pouvoir qui est de plus soutenu par les politiciens corrompus de Yankton dont l'émissaire est le commissaire véreux Hugo Jarry. Trixie est la seule personne de la série qui ose s'opposer véritablement à George Hearst, le blessant à l'épaule d'un coup de revolver à bout portant qui aurait dû le tuer... L'arme qui lui servira est présente dès le premier épisode). Seth Bullock arrête Hearst et le traîne en prison à travers la rue centrale en le tenant par l'oreille, mais cet acte humiliant est dérisoire et ne sert qu'à attiser la violence vindicative de Hearst. Même Aunt Lou, sa cuisinière noire ne peut que souhaiter à voix basse son meurtre alors qu'elle le tient pour responsable de la mort de son fils.

Les femmes sont les plus souvent les victimes désignées de cette violence : exploitées sexuellement, martyrisées, sacrifiées, en proie à la maladie et à l'emprise des drogues. La saison deux en particulier introduit un personnage de maniaque sexuel psychopathe, Francis Wolcott, le géologue au service de Hearst qui, pour compenser son impuissance sexuelle, tue plusieurs prostituées en toute impunité avant d'être licencié par son patron parce que ce genre de comportement n'est pas bon pour les affaires.

George Hearst, lui, souffre du dos. On le voit à plusieurs reprises allongé sur son lit ou à même le sol et il accepte volontiers les soins que lui prodigue John Langrishe, directeur d'une compagnie théâtrale dont l'attitude est ici ambiguë puisqu'il est normalement du côté de Swearengen. Parallèlement, la sexualité de Hearst est quasiment gommée, ce qui ne va pas de soi dans une ville regorgeant de lieux de plaisirs et où le corps des femmes s'obtient à vil prix. L'unique mention de son désir sexuel survient lorsque, après s'être emporté contre Alma Garrett (qui refuse de lui céder sa mine), Hearst reconnaît avoir eu envie de violer celle qui a tenté de lui résister en lui faisant une offre infamante pour lui et qu'il assimile à une tentative d'émasculation : " make of me a capon ». La transaction commerciale est ainsi associée à une métaphore sexuelle. Hearst ne supporte pas non plus la flatterie et la veulerie de Hugo Jarry, qu'il soupçonne (à tort) en évoquant Socrate et Alcibiade, de lui proposer une relation homosexuelle.

Le personnage d'Alma illustre aussi un rapport particulier au corps et à la maladie par sa neurasthénie et sa dépendance à la drogue (laudanum) qu'on la voit absorber dès sa première entrée en scène et de manière récurrente ensuite. Son pourvoyeur est Leon, employé servile de Cy Tolliver et qui apparaît aussi comme dépendant de l'opium fourni en gros par Wu, représentant des Tong chinois. Les prostituées de la Bella Union sont aussi victimes du fléau 
(conformément à la réalité historique selon David Milch5). Cette mise en relief du pathologique est également très prégnante dans la série. On peut évoquer, outre les calculs rénaux, l'épilepsie du pasteur, le révérend Smith, puis sa tumeur cérébrale qui le conduit à des comportements et des discours de plus en plus aberrants jusqu'à ce que Swearengen dont le frère est épileptique, le libère de ses souffrances en pratiquant une sorte d'euthanasie un peu brutale (il l'étouffe sous ses couvertures). L'épidémie de variole (smallpox) qui frappe le campement et cause de nombreuses victimes dont on voit le visage couvert de bubons, déformé par les douleurs dorsales, permet de montrer une gamme variée de comportements. Cy Tolliver, le plus cynique, abandonne à son sort Andy Cramed, son ex-partenaire de jeu quand il le croit atteint des premiers symptômes et le fait transporter en pleine forêt. Il sera sauvé par Calamity et deviendra pasteur, reniant sa vie d'avant la maladie. Par contre, outre Doc qui ne ménage pas son énergie, Jane se révèle totalement dévouée, passant tout son temps au chevet des malades. L'épidémie sert ainsi de révélateur des qualités humaines ou de catalyseur des énergies. Cochran lui-même, atteint de tuberculose, n'est pas épargné, son corps frêle secoué par des quintes de toux de plus en plus violentes à mesure que la maladie progresse, mais il ne meurt pas dans le cadre de série et c'est lui qui extrait de mauvaise grâce la balle de l'épaule de Hearst, ne bandant la plaie ouverte qu'à la demande expresse du magnat.

La série témoigne également de la volonté d'inscrire le corps dans un mode de figuration grotesque qui en mettant l'accent sur l'exagération ou la distorsion des traits physiques, privilégie une esthétique de la laideur, à la limite parfois du monstrueux. Les réalisateurs proposent une galerie de trognes et de corps grotesques comme celui du capitaine Turner déjà cité ou encore celui de Mose Manuel, prospecteur au comportement primaire dont la trogne grimaçante au crâne rasé semble petite, juchée sur un corps massif et gras, " a tub of blubber and guts » selon Jane. Cependant même ce personnage a priori sans foi ni loi qui tue son frère pour vendre sa concession, expérimente une forme de rédemption après avoir échappé de peu à la mort. Il devient l'ange gardien de Joanie Stubbs, traumatisée par la série mortifère qui frappe sa maison close, "Chez Ami », et subit une complète métamorphose soulignée par le jeu beaucoup plus sobre de l'acteur qui renonce aux grimaces et aux rictus divers. Il faudrait encore citer dans cette galerie de grotesques Richardson, le cuisinier de l'hôtel tenu par EB Farnum, les cheveux blancs, longs et en désordre, qui entretient un rapport animiste et fétichiste avec les bois de cerf qui ornent un des murs de l'hôtel. Farnum lui-même incarne une forme de grotesque déplaisant. Vêtu

5 Voir entretien avec David Milch, dans The Real Deadwood, documentaire inclus dans bonus du DVD. 
avec une élégance surannée un peu ridicule, soucieux de la moindre poussière sur son costume qu'il époussette à tout moment, Farnum est aussi l'homme aux mains moites et au comportement furtif de rongeur (il est souvent associé à ce type d'animal), image de la soumission servile au pouvoir, qu'il soit incarné par Swearengen ou Hearst. Le grotesque est ici renforcé par l'animalisation métaphorique. Ainsi, Jack McCall, après le meurtre de Wild Bill est enfermé avec les porcs, les hommes exhalent des odeurs de putois.

Certains organes corporels sont associés métaphoriquement. Si le pasteur Smith célèbre en citant la bible, le caractère indissociable et solidaire des parties du corps, Wild Bill reproche à Jack Mc Call son futur meurtrier d'avoir une paupière qui lui fait penser à un sexe féminin : "It looks like the hood on a cunt ». Plus tard, confirmant l'insulte, il évoque « his cunt-like mouth », s'attirant inévitablement la haine de son partenaire de poker qu'il humilie encore en lui donnant un dollar pour son repas.

Le personnage de Calamity, ivrognesse invétérée et vitupérante, n'est pas exempt d'exagération grotesque. Il associe certains aspects féminins (larmes, forme d'amour maternel avec l'enfant sauvée, vocation d'infirmière dévouée, sentiment amoureux informulé envers Hickok) et des traits plus typiquement masculins. Jane, vêtue comme un cow-boy présente en effet une forme de virilité provocante comme l'atteste cette apostrophe face à un parterre masculin qui s'esclaffe :

«I AM going now! Even without Bill. Even without Charlie. I know the road to Spearfish, and I don't drink when I am the only fucking one with balls! ».

À plusieurs reprises, elle exprime son agressivité face aux hommes, même vis à vis de ses proches comme Charlie Utter qu'elle accable d'insultes. Cependant ses tendances homosexuelles refoulées finissent par s'exprimer dans sa relation affective tourmentée avec Joannie dans la saison trois. Cette caractérisation grotesque est accentuée par le langage, Jane étant définie comme " sewermouth », qui ne cesse d'émettre des propos orduriers (comme son homologue dans l'Histoire), mais aussi par le jeu de l'actrice dont le corps est constamment en déséquilibre, en suspens entre deux positions.

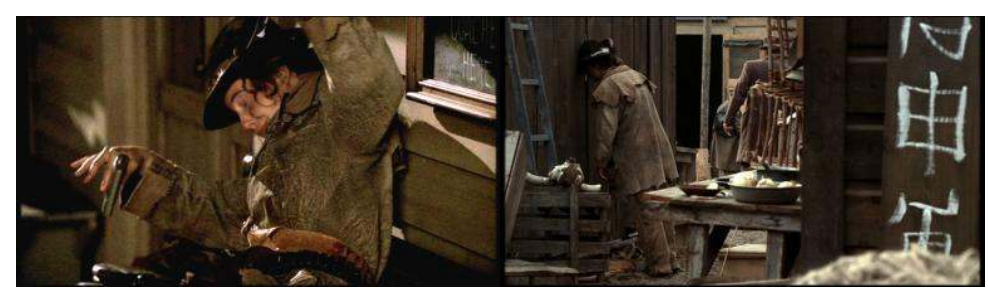


Cependant Milch et les divers réalisateurs proposent aussi d'autres types de relations physiques ou affectives, parfois non dénuées de tendresse. L'émotion se manifeste en particulier au moment de la mort du petit William (fils du frère de Seth qui a épousé sa veuve), tué par un coup de sabot de cheval sauvage. Toute la communauté se soucie du sort de l'enfant, prospecteurs ou commerçants autant que prostituées. Même les plus cyniques comme Wolcott semblent concernés. L'enterrement donne lieu à un recueillement collectif quasi unique dans la série, sans aucun débordement d'aucune sorte. La scène est mise en valeur sobrement. Elle est filmée comme un bloc autonome et n'est pas montée en parallèle avec d'autres scènes comme la séquence du mariage d'Alma et Ellsworth. L'attention du spectateur est concentrée sur le lieu de la cérémonie qui se déroule en plein air devant la maison nouvellement construite des Bullock et en présence d'une bonne partie de la communauté. Seul Swearengen s'abstient comme en témoignent les uniques plans où la caméra s'éloigne pour le filmer en observateur lointain sur son balcon. Autre moment chargé d'émotion, la scène où Emma, sous l'emprise du laudanum, tente de séduire son mari Ellsworth et l'embrasse sur la bouche. Le prospecteur la repousse avec délicatesse et décide de se séparer d'elle afin de l'aider à se libérer de la l'emprise de la drogue.

Le corps est également dramatisé, mis en relief par sa relation privilégiée au langage. Les dialogues très travaillés ont recours à de nombreuses métaphores liées au corps et à l'organique. La série a été remarquée pour l'emploi répété de mots grossiers, voire orduriers, relevant d'un lexique fortement sexualisé et parfois scatologique et abject. Tous les personnages, y compris les femmes (pas seulement Jane ou Trixie) ont recours à un langage coloré et sexualisé. Les mots les plus récurrents sont «fuck» ou «fucking » dont l'emploi est systématique chez $\mathrm{Al}$, mais fréquent dans la bouche de nombreux autres personnages, ou encore "cocksucker» (le seul mot anglais proféré par Wu qui lui attribue de ce fait une polysémie remarquable). Ce vocable qui évoque clairement une activité essentielle dans Deadwood (et pour $\mathrm{Al}$ ) est aussi une insulte qui concerne aussi bien les traîtres, les rivaux en affaires, les clients indélicats et les gêneurs de tout poil que les indiens définis comme «dirt worshipping cocksuckers », les noirs ou les chinois. D’autres mots réduisent le corps métonymiquement à un organe sexuel masculin («prick », « cock », "Johnson ») ou féminin ("pussy, "snatch», " cunt»). Diverse formulations abruptes définissent le corps objet ou la transaction sexuelle, contribuant ainsi à la réification de la femme, bousculée, culbutée, violentée, assassinée : "piece of pussy », "pussy is halfprice ", «branded snatch », " fucking cunt-struck» (Al s'exprimant sur le sentiment amoureux de Bullock!) etc. Au-delà de ce réseau lexical spécifique, la métaphore organique sert à caractériser la communauté elle-même définie comme un corps qui doit s'unir pour 
lutter contre la bureaucratie extérieure, celle des politiciens corrompus de Yankton. Comme le dit Erica Hill :

This struggle becomes Deadwood's largest body crisis of all, with the camp itself as a body, its thoroughfare veins, its residents blood, its center (the Gem) a heart ${ }^{6}$

Si le langage participe à l'évidence d'un « effet de réel » qui joue à plein de la métaphore corporelle, il contribue aussi à théâtraliser certaines scènes par l'emploi de dialogues qui relèvent à l'inverse d'une langue littéraire très métaphorique, voire poétique et enrichie par des emprunts shakespeariens et victoriens, en particulier dans les monologues et les apartés. Ainsi, c'est la parole qui redonne une forme de noblesse ou un sens du tragique à des personnages dont le corps est souvent trivialisé, animalisé, réifié. À l'évidence, la représentation de l'organique, tant verbale que visuelle, participe du réalisme souvent cru de la série. Le corps est utilisé à des fins diverses dans l'univers diégétique: force de travail, objet du désir ou support de fantasme, enjeu affectif ou financier. Il est aussi dramatisé, voire théâtralisé, dans un registre spectaculaire ou intime, public ou privé. Le corps apparaît prioritairement sous des dehors négatifs ou privatifs, corps souffrant, violenté, martyrisé, malade, infirme, dépendant de l'alcool, de la drogue, corseté par les vêtements, régi par les pulsions sexuelles, réduit à l'état de chair, viande, voire nourriture pour des porcs avides. Aucun personnage, victime, agent ou simple spectateur, n'est épargné par la souffrance ou la perte, même l'un des plus positifs, Martha Bullock qui doit faire le deuil de son fils et devient institutrice. La communauté se construit sur différentes formes de barbarie et de cruauté. L'affrontement spectaculaire attendu entre les deux camps n'a finalement pas lieu. Alors qu'une forme de pouvoir légal se met en place, les élections sont d'emblée truquées et Seth Bullock n'est pas élu au poste de sheriff, même s'il continue jusqu'au bout à exercer sa fonction face à Hearst en particulier.

La série se termine sur une ultime référence au corps absent de Jen la jeune prostituée sacrifiée. Al Swearengen essuie de nouveau frénétiquement la large tache de sang résiduelle qui souille son plancher. Le film se clôt sur ce plan trivial mais hautement symbolique. Cette fin dédramatisée a un caractère " domestique » qui suggère que Swearengen appartient à la communauté et s'intéresse à son sort et à son avenir. À l'inverse, Hearst n'appartient pas à la communauté. Après avoir vérifié l'état du corps dans le cercueil, il essuie sa botte pour en enlever les traces de sang, mais laisse le plancher maculé. Peu après il quitte Deadwood, ayant obtenu ce qu'il souhaitait, le rachat de

${ }^{6}$ Erica Hill, "'What's afflictin' you ? ‘: Corporeality, Body Crisis and the Body Politic in Deadwood ", in Reading Deadwood : a Western to Swear by, éd. David Lavery, Londres et New York, I.B. Tauris, 2006 
la mine d'Alma Garrett, à la recherche de nouveaux filons d'or, la «couleur » qui l'obsède et qui marque les corps et les âmes dans Deadwood. 